\title{
Ruelle type $L$-functions versus determinants of Laplacians for torsion free abelian groups*
}

\author{
Nobushige KUROKAWA, Masato WAKAYAMA and \\ Yoshinori YAMASAKI
}

August 2, 2018

\begin{abstract}
We study Ruelle's type zeta and $L$-functions for a torsion free abelian group $\Gamma$ of rank $\nu \geq 2$ defined via an Euler product. It is shown that the imaginary axis is a natural boundary of this zeta function when $\nu=2,4$ and 8 , and in particular, such a zeta function has no determinant expression. Thus, conversely, expressions like Euler's product for the determinant of the Laplacians of the torus $\mathbb{R}^{\nu} / \Gamma$ defined via zeta regularizations are investigated. Also, the limit behavior of an arithmetic function arising from the Ruelle type zeta function is observed.
\end{abstract}

2000 Mathematics Subject Classification : Primary 11M36, 11N37

Key words and phrases: Ruelle zeta function, Möbius inversion formula, Laplacian, Poisson summation formula, natural boundary, zeta regularization, $\zeta(3)$.

\section{Introduction}

The Ruelle zeta function is a particular kind of dynamical zeta function which counts periodic orbits for maps or flows. It was introduced by Ruelle in the mid-1970s [Ru]. The Ruelle zeta function is also regarded (essentially) as the Selberg zeta function when one considers the primitive geodesic flows on a hyperbolic space.

Instead, we have introduced in [KW3] various Ruelle type zeta functions for a complex torus and studied asymptotic averages of certain arithmetic functions arising from the logarithm of such Ruelle type zeta functions. In this paper, we study analytic properties of a Ruelle type $L$-function for a general torsion free abelian group and calculate also the determinant of the Laplacian for the corresponding group.

Let $\Gamma$ be a torsion free abelian group of rank $\nu(\nu \geq 2)$. Let $\tau_{1}, \ldots, \tau_{\nu}$ be a generator of $\Gamma$. Then we may write $\Gamma=\mathbb{Z} \tau_{1} \oplus \cdots \oplus \mathbb{Z} \tau_{\nu}$. A non-zero element $P=\sum_{j=1}^{\nu} n_{j} \tau_{j} \in \Gamma$ is said to be primitive if the integers $n_{j}$ are relatively prime, that is, the greatest common divisor $\operatorname{gcd}\left(n_{1}, \ldots, n_{\nu}\right)$ is equal to 1 . Here we interpret $\operatorname{gcd}\left(n_{1}, \ldots, n_{\nu}\right)$ as the greatest common divisor of the all positive factors of $\left|n_{j}\right|(j=1,2, \ldots, \nu)$. We denote by Prim $(\Gamma)$ the set of all primitive elements in $\Gamma$. Let $\ell: \Gamma \rightarrow \mathbb{R}_{\geq 0}$ be a function satisfying the homogeneity condition $\ell(j \gamma)=j \ell(\gamma)$ for any $j \in \mathbb{N}$ and $\gamma \in \Gamma$. For each element $\gamma \in \Gamma$ we define the norm $N(\gamma)$ by $N(\gamma)=e^{\ell(\gamma)}$. Note that any element $\gamma=\sum_{j=1}^{\nu} n_{j} \tau_{j} \in \Gamma$ can be uniquely expressed as $\gamma=d P$, where $d=\operatorname{gcd}\left(n_{1}, \ldots, n_{\nu}\right)$ and $P=P_{\gamma} \in \operatorname{Prim}(\Gamma)$. It is clear that $N(\gamma)=N\left(P_{\gamma}\right)^{d}$. Further, let $\rho: \Gamma \rightarrow U(N)$ be an $N$-dimensional unitary representation of $\Gamma$.

*Partially supported by Grant-in-Aid for Scientific Research (B) No.15340012 
For a given such norm function $N(\gamma)$ (or the length function $\ell(\gamma)$ ) and a representation $\rho$ of $\Gamma$, we introduce a Ruelle type $L$-function $L_{\Gamma}(s, \rho)$ for $\Gamma$ by the Euler product as

$$
L_{\Gamma}(s, \rho)=L_{\Gamma}(s, \rho ; \nu):=\prod_{P \in \operatorname{Prim}(\Gamma)} \operatorname{det}\left(1-\rho(P) N(P)^{-s}\right)^{-1} .
$$

When $\rho$ is the trivial representation of $\Gamma$, we write $\zeta_{\Gamma}(s ; \nu)=L_{\Gamma}(s$, the trivial rep.; $\nu)$ and call it a Ruelle type zeta function for $\Gamma$. Since $\Gamma$ is abelian, any irreducible unitary representation of $\Gamma$ is one dimensional. Thus, if we write the irreducible decomposition of $\rho$ as $\rho=\chi_{1} \oplus \cdots \oplus \chi_{N}$ (here we are taking account the multiplicity in the decomposition) where $\chi_{j}$ are one dimensional characters of $\Gamma$, we easily see that $L_{\Gamma}(s, \rho)=\prod_{P \in \operatorname{Prim}(\Gamma)} \prod_{j=1}^{N}\left(1-\chi_{j}(P) N(P)^{-s}\right)^{-1}=\prod_{j=1}^{N} L_{\Gamma}\left(s, \chi_{j}\right)$. In view of this decomposition, it is enough to treat the case where $\rho$ is one dimensional in our analysis.

The aim of the present paper is to show first that, for $\nu=2,4$ or 8 , the Ruelle type zeta function $\zeta_{\Gamma}(s ; \nu)$ has a natural boundary $\operatorname{Re}(s)=0$ when $\ell(\gamma)$ is defined by the usual Euclidean norm (Theorem 2.2). This shows, in particular, that $\operatorname{such} \zeta_{\Gamma}(s ; \nu)$ does not have a determinant expression for the Laplacian of the torus $\mathbb{R}^{\nu} / \Gamma$ (here the determinant is defined by the zeta regularization. See $\S 3)$. Thus, conversely, we study the determinant $\operatorname{det}\left(\Delta+s^{2}\right)$ of the Laplacian $\Delta$ on the torus and show that it has a sort of Euler products (Theorem 3.1). Though the results show that $\operatorname{det}\left(\Delta+s^{2}\right)$ can not give $\zeta_{\Gamma}(s ; \nu)$, we notice that the logarithm of both the functions involve certain common arithmetic function (compare Corollary 3.6 with (4.2)). Therefore, we also discuss asymptotic averages of certain arithmetic functions arising from these Ruelle type zeta functions like in [KW3].

\section{$2 \quad L_{\sqrt{+^{2}}}(s, \chi)$}

We study a Ruelle type $L$-function $L_{\Gamma}(s, \rho ; \nu)$ when $\ell(\gamma)=\sqrt{n_{1}^{2}+\cdots+n_{\nu}^{2}}$ for $\gamma=\sum_{j=1}^{\nu} n_{j} \tau_{j} \in \Gamma$. To distinguish the case from a general choice of length functions, we write $L_{\sqrt{+^{2}}}(s, \rho ; \nu)$ instead of $L_{\Gamma}(s, \rho ; \nu)$. Let $\chi$ be a unitary character of $\Gamma=\mathbb{Z} \tau_{1} \oplus \cdots \oplus \mathbb{Z} \tau_{\nu}$. Put $\chi\left(\tau_{j}\right)=e^{2 \pi i \alpha_{j}}$ for $j=1, \ldots, \nu$. Then we have $\chi\left(\sum_{j=1}^{\nu} n_{j} \tau_{j}\right)=e^{2 \pi i\left(n_{1} \alpha_{1}+\cdots+n_{\nu} \alpha_{\nu}\right)}$. To economize the space, we use a multi-index. Write an element $\gamma=\sum_{j=1}^{\nu} n_{j} \tau_{j} \in \Gamma$ in a general position as $\gamma=\gamma\left(n_{1}, \ldots, n_{\nu}\right)=\gamma(\mathbf{n})$. In general, for $\mathbf{x}, \mathbf{y} \in \mathbb{R}^{\nu}$ we put $\mathbf{x y}:=\sum_{j=1}^{\nu} x_{j} y_{j}$ and put $|\mathbf{x}|:=\sqrt{\mathbf{x x}}=\sqrt{x_{1}^{2}+\cdots+x_{\nu}{ }^{2}}$. Hence $\ell(\gamma(\mathbf{n}))=|\mathbf{n}|$. We may therefore write $\chi(\gamma(\mathbf{n}))=e^{2 \pi i \mathbf{n} \boldsymbol{\alpha}}$ for some $\boldsymbol{\alpha}=\left(\alpha_{1}, \ldots, \alpha_{\nu}\right) \in \mathbb{R}^{\nu} / \mathbb{Z}^{\nu}$. Thus we sometimes identify the unitary character $\chi$ of $\Gamma\left(\cong \mathbb{Z}^{\nu}\right)$ with $\boldsymbol{\alpha} \in \mathbb{R}^{\nu} / \mathbb{Z}^{\nu}\left(\cong \hat{\mathbb{Z}}^{\nu}\right)$ and write the Ruelle type $L$-function $L_{\sqrt{+^{2}}}(s, \chi)$ as $L_{\sqrt{+^{2}}}(s, \boldsymbol{\alpha})$ :

$$
L_{\sqrt{+^{2}}}(s, \boldsymbol{\alpha})=L_{\sqrt{+^{2}}}(s, \boldsymbol{\alpha} ; \nu):=\prod_{\operatorname{gcd} \mathbf{p}=1}\left(1-e^{2 \pi i \mathbf{p} \boldsymbol{\alpha}} e^{-s|\mathbf{p}|}\right)^{-1} .
$$

It is easy to see that the Euler product (2.1) converges absolutely for $\operatorname{Re}(s)>0$ because $\nu|\mathbf{x}| \geq$ $\left|x_{1}\right|+\cdots+\left|x_{\nu}\right|$ for $\mathbf{x} \in \mathbb{R}^{\nu}$, whence it defines a holomorphic function for $\operatorname{Re}(s)>0$. Also, we notice that $L_{\sqrt{+^{2}}}(s, \boldsymbol{\alpha}(\varepsilon))=L_{\sqrt{+^{2}}}(s, \boldsymbol{\alpha})$ where $\boldsymbol{\alpha}(\varepsilon)=\left(\varepsilon_{1} \alpha_{1}, \ldots, \varepsilon_{\nu} \alpha_{\nu}\right)$ for $\varepsilon=\left(\varepsilon_{1}, \ldots, \varepsilon_{\nu}\right) \in\{ \pm\}^{\nu}$ and in particular, $L_{\sqrt{+^{2}}}(s, \bar{\chi})=L_{\sqrt{+^{2}}}(s, \chi)$.

We first show the

Proposition 2.1. The logarithmic derivative of $L_{\sqrt{+^{2}}}(s, \boldsymbol{\alpha} ; \nu)$ is holomorphic in $\operatorname{Re}(s)>0$ and has the expression

$$
\frac{L_{\sqrt{+^{2}}}^{\prime}}{L_{\sqrt{+^{2}}}}(s, \boldsymbol{\alpha} ; \nu)=2(2 \sqrt{\pi})^{\nu-1} \Gamma\left(\frac{\nu+1}{2}\right) \sum_{n=1}^{\infty} \frac{\gamma(n)}{n^{\nu+1}} \sum_{\mathbf{m} \in \mathbb{Z}^{\nu}} \frac{(2 \pi|\mathbf{m} / n+\boldsymbol{\alpha}|)^{2}-\nu s^{2}}{\left\{s^{2}+(2 \pi|\mathbf{m} / n+\boldsymbol{\alpha}|)^{2}\right\}^{\frac{\nu+3}{2}}}
$$


for $\operatorname{Re}(s)>0$. Here $\gamma(n)=\prod_{p \mid n}(1-p)$.

Proof. The first assertion is clear, because $L_{\sqrt{+^{2}}}(s, \boldsymbol{\alpha})$ is holomorphic and non-zero in $\operatorname{Re}(s)>0$. We introduce now an auxiliary function $G(s, \boldsymbol{\alpha})$ by

$$
G(s, \boldsymbol{\alpha}):=\prod_{\mathbf{n} \in \mathbb{Z}^{\nu} \backslash\{\mathbf{0}\}}\left(1-e^{2 \pi i \mathbf{n} \boldsymbol{\alpha}} e^{-s|\mathbf{n}|}\right)^{-1} .
$$

Then we have

$$
\log G(s, \boldsymbol{\alpha})=-\sum_{\mathbf{n} \in \mathbb{Z}^{\nu} \backslash\{\mathbf{0}\}} \log \left(1-e^{2 \pi i \mathbf{n} \boldsymbol{\alpha}} e^{-s|\mathbf{n}|}\right)=\sum_{\mathbf{n} \in \mathbb{Z}^{\nu} \backslash\{\mathbf{0}\}} \sum_{\ell=1}^{\infty} \frac{1}{\ell} e^{2 \pi i \ell \mathbf{n} \boldsymbol{\alpha}} e^{-s \ell|\mathbf{n}|} .
$$

It is immediate to see that

$$
\log G(s, \boldsymbol{\alpha})=\sum_{d=1}^{\infty} \sum_{\operatorname{gcd} \mathbf{p}=1} \sum_{\ell=1}^{\infty} \frac{e^{2 \pi i \ell(d \mathbf{p}) \boldsymbol{\alpha}} e^{-s \ell|d \mathbf{p}|}}{\ell}=\sum_{d=1}^{\infty} \log L_{\sqrt{+^{2}}}(d s, d \boldsymbol{\alpha}) .
$$

Hence the Möbius inversion formula yields

$$
\log L_{\sqrt{+^{2}}}(s, \boldsymbol{\alpha})=\sum_{m=1}^{\infty} \mu(m) \log G(m s, m \boldsymbol{\alpha}) .
$$

Here $\mu(n)$ denotes the Möbius function defined as

$$
\mu(n)= \begin{cases}1 & \text { if } n=1 \\ (-1)^{k} & \text { if } n \text { is a product of } k \text { distinct primes } \\ 0 & \text { otherwise. }\end{cases}
$$

In fact, since $\sum_{m \mid n} \mu(m)=\delta_{n 1}$, we see that

$$
\begin{aligned}
\sum_{m=1}^{\infty} \mu(m) \log G(m s, m \boldsymbol{\alpha}) & =\sum_{m=1}^{\infty} \mu(m) \sum_{d=1}^{\infty} \log L_{\sqrt{+^{2}}}(d m s, d m \boldsymbol{\alpha}) \\
& =\sum_{n=1}^{\infty}\left\{\sum_{m \mid n} \mu(m)\right\} \log L_{\sqrt{+^{2}}}(n s, n \boldsymbol{\alpha})=\log L_{\sqrt{+^{2}}}(s, \boldsymbol{\alpha})
\end{aligned}
$$

We now put

$$
g(s, \boldsymbol{\alpha}):=\sum_{\mathbf{n} \in \mathbb{Z}^{\nu} \backslash\{\mathbf{0}\}} e^{2 \pi i \mathbf{n} \boldsymbol{\alpha}} e^{-s|\mathbf{n}|} .
$$

Then by (2.3) we easily get the relation

$$
\log G(s, \boldsymbol{\alpha})=\sum_{\ell=1}^{\infty} \frac{1}{\ell} g(\ell s, \ell \boldsymbol{\alpha})
$$

Let $f(\mathbf{x})$ be a smooth rapidly decreasing function on $\mathbb{R}^{\nu}$. Write the Fourier transform of $f(\mathbf{x})$ by $\hat{f}(\mathbf{y})=\int_{\mathbb{R}^{\nu}} f(\mathbf{x}) e^{2 \pi i \mathbf{y} \mathbf{x}} d \mathbf{x}$. It can be calculated (see, e.g., $[\mathrm{Mu}]$ ) as

$$
\left(e^{-2 \pi t|\mathbf{x}|} e^{-2 \pi i \mathbf{z x}}\right)^{\wedge}(\mathbf{y})=\frac{2 t}{\operatorname{Area}\left(S^{\nu}\right)} \cdot\left(t^{2}+|\mathbf{y}-\mathbf{z}|^{2}\right)^{-\frac{\nu+1}{2}} \quad(\operatorname{Re}(t)>0),
$$


where Area $\left(S^{\nu}\right)=2 \pi^{\frac{\nu+1}{2}} / \Gamma\left(\frac{\nu+1}{2}\right)$. Therfore, the Poisson summation formula applying to the function $e^{-2 \pi t|\mathbf{x}|} e^{2 \pi i \boldsymbol{\alpha} \mathbf{x}}$ reads

$$
\sum_{\mathbf{n} \in \mathbb{Z}^{\nu}} e^{-2 \pi t|\mathbf{n}|} e^{-2 \pi i \mathbf{z n}}=\frac{2 t}{\operatorname{Area}\left(S^{\nu}\right)} \sum_{\mathbf{m} \in \mathbb{Z}^{\nu}}\left(t^{2}+|\mathbf{m}-\mathbf{z}|^{2}\right)^{-\frac{\nu+1}{2}} .
$$

This shows that for $\operatorname{Re}(s)>0$ we have

$$
g(s, \boldsymbol{\alpha})+1=\frac{2(2 \pi)^{\nu} s}{\operatorname{Area}\left(S^{\nu}\right)} \sum_{\mathbf{m} \in \mathbb{Z}^{\nu}}\left\{s^{2}+(2 \pi|\mathbf{m}+\boldsymbol{\alpha}|)^{2}\right\}^{-\frac{\nu+1}{2}} .
$$

Differentiating this equation with respect to $s$, we obtain

$$
g^{\prime}(s, \boldsymbol{\alpha})=\frac{2(2 \pi)^{\nu}}{\operatorname{Area}\left(S^{\nu}\right)} \sum_{\mathbf{m} \in \mathbb{Z}^{\nu}} \frac{(2 \pi|\mathbf{m}+\boldsymbol{\alpha}|)^{2}-\nu s^{2}}{\left\{s^{2}+(2 \pi|\mathbf{m}+\boldsymbol{\alpha}|)^{2}\right\}^{\frac{\nu+3}{2}}} .
$$

By the relations (2.4) and (2.5), we have

$$
\frac{L_{\sqrt{+^{2}}}^{\prime}}{L_{\sqrt{+^{2}}}}(s, \boldsymbol{\alpha})=\sum_{m=1}^{\infty} m \mu(m) \frac{G^{\prime}}{G}(m s, m \boldsymbol{\alpha}), \quad \frac{G^{\prime}}{G}(s, \boldsymbol{\alpha})=\sum_{\ell=1}^{\infty} g^{\prime}(\ell s, \ell \boldsymbol{\alpha}) .
$$

From these equations it follows that

$$
\frac{L_{\sqrt{+^{2}}}^{\prime}}{L_{\sqrt{+^{2}}}}(s, \boldsymbol{\alpha})=\sum_{m=1}^{\infty} \sum_{\ell=1}^{\infty} m \mu(m) g^{\prime}(m \ell s, m \ell \boldsymbol{\alpha})=\sum_{n=1}^{\infty} \gamma(n) g^{\prime}(n s, n \boldsymbol{\alpha}) .
$$

Here we have used an easily verified formula $\sum_{m \mid n} m \mu(m)=\gamma(n)$. Hence, it follows from (2.7) and (2.8) that

$$
\begin{aligned}
\frac{L_{\sqrt{+^{2}}}^{\prime}}{L_{\sqrt{+^{2}}}}(s, \boldsymbol{\alpha}) & =\frac{2(2 \pi)^{\nu}}{\operatorname{Area}\left(S^{\nu}\right)} \sum_{n=1}^{\infty} \gamma(n) \sum_{\mathbf{m} \in \mathbb{Z}^{\nu}} \frac{(2 \pi|\mathbf{m}+n \boldsymbol{\alpha}|)^{2}-\nu(n s)^{2}}{\left\{(n s)^{2}+(2 \pi|\mathbf{m}+n \boldsymbol{\alpha}|)^{2}\right\}^{\frac{\nu+3}{2}}} \\
& =2(2 \sqrt{\pi})^{\nu-1} \Gamma\left(\frac{\nu+1}{2}\right) \sum_{n=1}^{\infty} \frac{\gamma(n)}{n^{\nu+1}} \sum_{\mathbf{m} \in \mathbb{Z}^{\nu}} \frac{(2 \pi|\mathbf{m} / n+\boldsymbol{\alpha}|)^{2}-\nu s^{2}}{\left\{s^{2}+(2 \pi|\mathbf{m} / n+\boldsymbol{\alpha}|)^{2}\right\}^{\frac{\nu+3}{2}}}
\end{aligned}
$$

This shows the proposition.

Put

$$
\begin{aligned}
C(\nu) & :=2(2 \sqrt{\pi})^{\nu-1} \Gamma\left(\frac{\nu+1}{2}\right), \\
\Phi(s, \boldsymbol{\alpha}, t ; \nu) & :=\sum_{n \in \mathbb{N}, \mathbf{m} \in \mathbb{Z}^{\nu}} \frac{\gamma(n)}{n^{\nu+1}}\left(s^{2}+(2 \pi|\mathbf{m} / n+\boldsymbol{\alpha}|)^{2}\right)^{-t} .
\end{aligned}
$$

Then the formula (2.2) can be written as

$$
\frac{L_{\sqrt{t^{2}}}^{\prime}}{L_{\sqrt{+^{2}}}}(s, \boldsymbol{\alpha} ; \nu)=C(\nu)\left(\Phi\left(s, \boldsymbol{\alpha}, \frac{\nu+1}{2} ; \nu\right)-(\nu+1) s^{2} \Phi\left(s, \boldsymbol{\alpha}, \frac{\nu+3}{2} ; \nu\right)\right) .
$$

The following theorem shows that $\zeta_{\sqrt{t^{2}}}(s ; \nu):=L_{\sqrt{t^{2}}}(s, \mathbf{0} ; \nu)$ can not be extended meromorphically across the imaginary axis $\operatorname{Re}(s)=0$ if $\nu=2,4$ or 8 , that is, the cases where the number $r_{\nu}(n)$ of expressions of a positive integer $n$ by a sum of $\nu$ integers square is essentially multiplicative. 
Theorem 2.2. Let $\nu=2,4$ or 8 . Then the imaginary axis is a natural boundary of $\zeta_{\sqrt{+^{2}}}(s ; \nu)$.

From the expression (2.9), to prove Theorem 2.2, it is sufficient to show that the imaginary axis $\operatorname{Re}(s)=0$ is a natural boundary of the function $\Phi(s, \mathbf{0}, t ; \nu)$. Let $\mathbb{S}_{\nu}:=\left\{\frac{|\mathbf{m}|}{n} \mid n \in \mathbb{N}, \mathbf{m} \in \mathbb{Z}^{\nu}\right\}$. For a given $x_{0} \in \mathbb{R}$, let us denote $R_{\nu}\left(x_{0}\right)$ as the coefficient of $\left(s^{2}+\left(2 \pi x_{0}\right)^{2}\right)^{-t}$ in the series $\Phi(s, \mathbf{0}, t ; \nu)$ :

$$
\Phi(s, \mathbf{0}, t ; \nu)=\sum_{x_{0} \in \mathbb{S}_{\nu}} R_{\nu}\left(x_{0}\right)\left(s^{2}+\left(2 \pi x_{0}\right)^{2}\right)^{-t}
$$

Note that $R_{\nu}\left(x_{0}\right) \equiv 0$ if $x_{0} \notin \mathbb{S}_{\nu}$

Lemma 2.3. Let $\widetilde{m}$ and $\widetilde{n}$ be positive integers satisfying $(\widetilde{m}, \widetilde{n})=1$. Then we have

$$
R_{\nu}\left(\frac{\widetilde{m}}{\widetilde{n}}\right)=\frac{1}{\widetilde{n}^{\nu+1}} \sum_{k=1}^{\infty} \frac{\gamma(k \widetilde{n}) r_{\nu}\left(k^{2} \widetilde{m}^{2}\right)}{k^{\nu+1}},
$$

where $r_{\nu}(n):=\#\left\{\left(m_{1}, \ldots, m_{\nu}\right) \in \mathbb{Z}^{\nu} \mid m_{1}^{2}+\cdots+m_{\nu}^{2}=n\right\}$.

Proof. Since $(\widetilde{m}, \widetilde{n})=1$, the term $\left(s^{2}+\left(2 \pi \frac{\widetilde{m}}{n}\right)^{2}\right)^{-t}$ appears in $\Phi(s, \mathbf{0}, t ; \nu)$ when $n$ and $\mathbf{m}$ can be written as $n=k \widetilde{n}$ and $|\mathbf{m}|=k \tilde{m}$ with some $k \in \mathbb{N}$. Therefore, one can calculate as

$$
\begin{aligned}
R_{\nu}\left(\frac{\widetilde{m}}{\widetilde{n}}\right) & =\sum_{k=1}^{\infty} \sum_{|\mathbf{m}|=k \widetilde{m}} \frac{\gamma(k \widetilde{n})}{(k \widetilde{n})^{\nu+1}}=\frac{1}{\widetilde{n}^{\nu+1}} \sum_{k=1}^{\infty} \sum_{\substack{m_{1}, \ldots, m_{\nu} \in \mathbb{Z} \\
m_{1}{ }^{2}+\cdots+m_{\nu}{ }^{2}=k^{2} \widetilde{m}^{2}}} \frac{\gamma(k \widetilde{n})}{k^{\nu+1}} \\
& =\frac{1}{\widetilde{n}^{\nu+1}} \sum_{k=1}^{\infty} \frac{\gamma(k \widetilde{n}) r_{\nu}\left(k^{2} \widetilde{m}^{2}\right)}{k^{\nu+1}} .
\end{aligned}
$$

This shows the assertion.

The following lemma is crucial for the proof of Theorem 2.2.

Lemma 2.4. Assume $\nu=2,4$ or 8 . For any prime $p$ and a non-negative integer $e$, it holds that

$$
\sum_{n=1}^{\infty} r_{\nu}\left(p^{2(n+e)}\right) p^{-n(\nu+1)} \neq \frac{r_{\nu}\left(p^{2 e}\right)}{p-1} .
$$

Proof. It is a classical result that the arithmetic function $\frac{1}{2 \nu} r_{\nu}(n)$ is multiplicative, that is, $\frac{1}{2 \nu} r_{\nu}(m n)=$ $\frac{1}{2 \nu} r_{\nu}(m) \frac{1}{2 \nu} r_{\nu}(n)$ for $(m, n)=1$ if and only if $\nu=2,4$ or 8 , and is explicitely given (by Jacobi when $\nu=4)$ as

$$
\frac{1}{2 \nu} r_{\nu}(n)= \begin{cases}\sum_{m \mid n} \chi_{-4}(m) & \text { if } \nu=2, \\ \sum_{m \mid n, 4 \nmid m} m & \text { if } \nu=4, \\ (-1)^{n} \sum_{m \mid n}(-1)^{m} m^{3} & \text { if } \nu=8,\end{cases}
$$

where $\chi_{-4}(n)$ is the primitive Dirichlet character modulo 4 (see, e.g., [BC]). We check the formula (2.11) for each $\nu$. 
1. The case $\nu=2$ : Let $\ell$ be a positive integer and $p$ a prime. From (2.12), it holds that

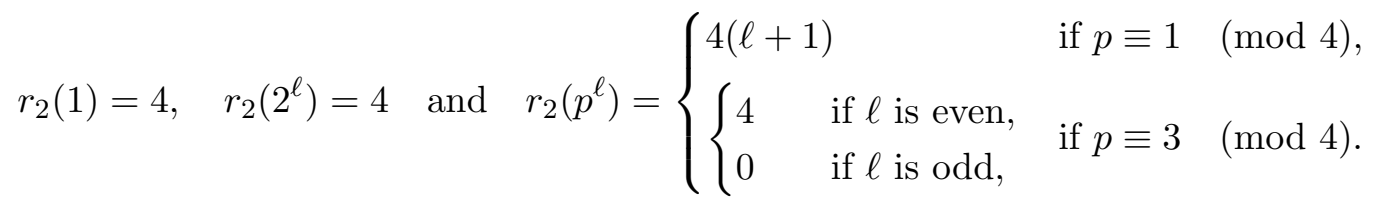

Therfore we have

(a) for $p=2$

$$
\begin{aligned}
& \text { L.H.S of }(2.11)=4 \sum_{n=1}^{\infty} 2^{-3 n}=\frac{4}{7}, \\
& \text { R.H.S of }(2.11)=4 .
\end{aligned}
$$

Hence the claim (2.11) is true.

(b) for $p \equiv 1(\bmod 4)$

$$
\begin{aligned}
& \text { L.H.S of }(2.11)=\sum_{n=1}^{\infty} 4(2(n+e)+1) 2^{-3 n}=\frac{8 p^{3}+4(2 e+1)\left(p^{3}-1\right)}{\left(p^{3}-1\right)^{2}} \\
& \text { R.H.S of }(2.11)=\frac{4(2 e+1)}{p-1}
\end{aligned}
$$

In this case, since $p^{\ell} \equiv 1(\bmod 4)$ for all $\ell \geq 1$, we obtain $(2.11)$.

(c) for $p \equiv 3(\bmod 4)$

$$
\begin{aligned}
& \text { L.H.S of }(2.11)=4 \sum_{n=1}^{\infty} p^{-3 n}=\frac{4}{p^{3}-1}, \\
& \text { R.H.S of }(2.11)=\frac{4}{p-1} .
\end{aligned}
$$

Hence (2.11) follows clearly.

2. The case $\nu=4$ : It follows from (2.12) again that

$$
r_{4}(1)=8, \quad r_{4}\left(2^{\ell}\right)=24 \quad \text { and } \quad r_{4}\left(p^{\ell}\right)=8 \cdot \frac{p^{\ell+1}-1}{p-1} \quad(p \neq 2) .
$$

Hence we have

(a) for $p=2$

$$
\begin{aligned}
& \text { L.H.S of }(2.11)=24 \sum_{n=1}^{\infty} 2^{-5 n}=\frac{24}{31}, \\
& \text { R.H.S of }(2.11)=\left\{\begin{array}{lll}
8 & \text { if } & e=0 \\
24 & \text { if } & e>0 .
\end{array}\right.
\end{aligned}
$$

This shows that (2.11) is true. 
(b) for $p \neq 2$

$$
\begin{aligned}
& \text { L.H.S of }(2.11)=8 \sum_{n=1}^{\infty} \frac{p^{2(n+e)+1}-1}{p-1} p^{-5 n}=\frac{8}{p-1}\left(\frac{p^{2 e+1}}{p^{3}-1}-\frac{1}{p^{5}-1}\right), \\
& \text { R.H.S of }(2.11)=\frac{8}{p-1} \cdot \frac{p^{2 e+1}-1}{p-1} .
\end{aligned}
$$

Since $p>3$, we have

$$
\frac{p^{2 e+1}-1}{p-1}-\left(\frac{p^{2 e+1}}{p^{3}-1}-\frac{1}{p^{5}-1}\right)>\frac{p^{2 e+1}-1}{p-1}-\frac{p^{2 e+1}}{p^{3}-1}>0 .
$$

Hence the claim (2.11) follows.

3. The case $\nu=8$ : We have

$$
r_{8}(1)=16, \quad r_{8}\left(2^{\ell}\right)=16 \cdot \frac{2^{3(\ell+1)}-15}{7} \quad \text { and } \quad r_{8}\left(p^{\ell}\right)=16 \cdot \frac{p^{3(\ell+1)}-1}{p^{3}-1} \quad(p \neq 2) .
$$

Hence it holds that

(a) for $p=2$

$$
\begin{aligned}
& \text { L.H.S of }(2.11)=16 \sum_{n=1}^{\infty} \frac{2^{3(2(n+e)+1)}-15}{7} 2^{-9 n}=\frac{16}{7}\left(\frac{2^{3(2 e+1)}}{7}-\frac{15}{511}\right), \\
& \text { R.H.S of }(2.11)= \begin{cases}16 & \text { if } e=0, \\
16 \cdot \frac{2^{3(2 e+1)}-15}{7} & \text { if } e>0 .\end{cases}
\end{aligned}
$$

Hence the claim (2.11) follows.

(b) for $p \neq 2$

$$
\begin{aligned}
& \text { L.H.S of }(2.11)=16 \sum_{n=1}^{\infty} \frac{p^{3(2(n+e)+1)}-1}{p^{3}-1} p^{-9 n}=\frac{16}{p^{3}-1}\left(\frac{p^{3(2 e+1)}}{p^{3}-1}-\frac{1}{p^{9}-1}\right), \\
& \text { R.H.S of }(2.11)=\frac{16}{p-1} \cdot \frac{p^{3(2 e+1)}-1}{p^{3}-1} .
\end{aligned}
$$

By the same argument in the cases of $\nu=4$ and $p \neq 2$, one can show (2.11).

This completes the proof of the lemma.

We now prove Theorem 2.2.

Proof of Theorem 2.2. Since $\mathbb{Q}$ is dense in $\mathbb{R}$, it is sufficient to show that $R_{\nu}(\xi) \neq 0$ for any positive $\xi \in \mathbb{Q}$ by the expression (2.10) of $\Phi(s, \boldsymbol{\alpha}, t ; \nu)$. Let $\widetilde{m}$ and $\widetilde{n}$ be positive integers satisfying $(\widetilde{m}, \widetilde{n})=1$. Let $\widetilde{m}=p_{1}{ }^{e_{1}} \cdots p_{s}{ }^{e_{s}}$ and $\widetilde{n}=q_{1} f_{1} \cdots q_{t}{ }^{f_{t}}$ be their prime factorizations. Note that $\left(p_{i}, q_{j}\right)=1$ for 
any $i$ and $j$. We shall prove $R_{\nu}\left(\frac{\widetilde{m}}{\tilde{n}}\right) \neq 0$. Since the functions $\gamma(n)$ and $\frac{1}{2 \nu} r_{\nu}(n)$ with $\nu=2,4$ or 8 are multiplicative, we have from Lemma 2.3 that

$$
\begin{aligned}
& \widetilde{n}^{\nu+1} R_{\nu}\left(\frac{\widetilde{m}}{\widetilde{n}}\right)=\sum_{\substack{m_{1}, \ldots, m_{t} \geq 0 \\
n_{1}, \ldots, n_{s} \geq 0}} \sum_{\substack{\left(k, p_{i}\right)=1 \\
\left(k, q_{j}\right)=1}} \frac{\gamma\left(k \prod_{j=1}^{t} q_{j}^{m_{j}+f_{j}} \prod_{i=1}^{s} p_{i}{ }^{n_{i}}\right) r_{n}\left(k^{2} \prod_{j=1}^{t} q_{j}^{2 m_{j}} \prod_{i=1}^{s} p_{i}{ }^{2\left(n_{i}+e_{i}\right)}\right)}{\left(k \prod_{j=1}^{t} q_{j}^{m_{j}} \prod_{i=1}^{s} p_{i} n^{n_{i}}\right)^{\nu+1}} \\
& =\left(\prod_{j=1}^{t} \frac{1-q_{j}}{2 \nu} \sum_{m_{j}=0}^{\infty} \frac{r_{\nu}\left(q_{j}^{2 m_{j}}\right)}{q_{j}^{m_{j}(\nu+1)}}\right)\left(\prod_{i=1}^{s} \frac{1}{2 \nu} \sum_{n_{i}=0}^{\infty} \frac{\gamma\left(p_{i}^{n_{i}}\right) r_{\nu}\left(p_{i}^{2\left(n_{i}+e_{i}\right)}\right)}{p_{i}^{n_{i}(\nu+1)}}\right)\left(\sum_{\substack{\left(k, p_{i}\right)=1 \\
\left(k, q_{j}\right)=1}} \frac{\gamma(k) r_{\nu}\left(k^{2}\right)}{k^{\nu+1}}\right) \\
& =\prod_{j=1}^{t} \frac{1-q_{j}}{2 \nu} E_{\nu, q_{j}}\left(\frac{\widetilde{m}}{\widetilde{n}}\right) \times \prod_{i=1}^{s} \frac{1}{2 \nu} F_{\nu, p_{i}}\left(\frac{\widetilde{m}}{\widetilde{n}}\right) \times G_{\nu}\left(\frac{\widetilde{m}}{\widetilde{n}}\right)
\end{aligned}
$$

where

$$
\begin{aligned}
E_{\nu, q_{j}}\left(\frac{\widetilde{m}}{\widetilde{n}}\right): & =\sum_{m_{j}=0}^{\infty} \frac{r_{\nu}\left(q_{j}^{2 m_{j}}\right)}{q_{j}^{m_{j}(\nu+1)}}, \quad F_{\nu, p_{i}}\left(\frac{\widetilde{m}}{\widetilde{n}}\right):=\sum_{n_{i}=0}^{\infty} \frac{\gamma\left(p_{i}^{\nu_{i}}\right) r_{\nu}\left(p_{i}^{2\left(n_{i}+e_{i}\right)}\right)}{p_{i} n_{i}(\nu+1)} \text { and } \\
G_{\nu}\left(\frac{\widetilde{m}}{\widetilde{n}}\right): & =\sum_{\substack{\left.k, p_{i}\right)=1 \\
\left(k, q_{j}\right)=1}} \frac{\gamma(k) r_{\nu}\left(k^{2}\right)}{k^{\nu+1}} .
\end{aligned}
$$

Note that $\gamma\left(q_{j}{ }^{m_{j}+f_{j}}\right)=\gamma\left(q_{j}\right)=1-q_{j}$, because $f_{j} \geq 1$. Since $E_{\nu, q_{j}}\left(\frac{\widetilde{m}}{n}\right) \neq 0$ for all $j$, it suffices to show that $F_{\nu, p_{i}}\left(\frac{\widetilde{m}}{\widetilde{n}}\right) \neq 0$ for all $i$ and $G_{\nu}\left(\frac{\widetilde{m}}{\widetilde{n}}\right) \neq 0$.

1. The function $F_{\nu, p_{i}}\left(\frac{\widetilde{m}}{\widetilde{n}}\right)$ : Since

$$
F_{\nu, p_{i}}\left(\frac{\widetilde{m}}{\widetilde{n}}\right)=r_{\nu}\left(p^{2 e_{i}}\right)-\left(p_{i}-1\right) \sum_{n_{i}=1}^{\infty} \frac{r_{\nu}\left(p_{i}{ }^{2\left(n_{i}+e_{i}\right)}\right)}{p_{i}^{n_{i}(\nu+1)}}
$$

we have

$$
F_{\nu, p_{i}}\left(\frac{\widetilde{m}}{\widetilde{n}}\right) \neq 0 \Longleftrightarrow \sum_{n_{i}=1}^{\infty} r_{\nu}\left(p_{i}{ }^{2\left(n_{i}+e_{i}\right)}\right) p_{i}{ }^{-n_{i}(\nu+1)} \neq \frac{r_{\nu}\left(p^{2 e_{i}}\right)}{p_{i}-1}
$$

By Lemma 2.4 (the case of $e=e_{i} \geq 1$ ), the right-above is true, whence the claim follows.

2. The function $G_{\nu}\left(\frac{\widetilde{m}}{n}\right)$ : Since $\gamma(n)$ and $\frac{1}{2 \nu} r_{\nu}\left(n^{2}\right)$ are multiplicative, the series

$$
G_{\nu}:=\sum_{k=1}^{\infty} \frac{\gamma(k) r_{\nu}\left(k^{2}\right)}{k^{\nu+1}}
$$

has an Euler product expression. In fact, we have $G_{\nu}=2 \nu \prod_{p \text { :prime }} G_{\nu, p}$, where

$$
G_{\nu, p}:=\frac{1}{2 \nu} \sum_{\ell=0}^{\infty} \gamma\left(p^{\ell}\right) r_{\nu}\left(p^{2 \ell}\right) p^{-\ell(\nu+1)}=1-\frac{p-1}{2 \nu} \sum_{\ell=1}^{\infty} r_{\nu}\left(p^{2 \ell}\right) p^{-\ell(\nu+1)} \neq 0 .
$$

The fact $G_{\nu, p} \neq 0$ is, in fact, derived from Lemma 2.4 (i.e., look at the case $e=0$ ). It follows that

$$
G_{\nu}\left(\frac{\widetilde{m}}{\widetilde{n}}\right)=\prod_{\substack{p: \text { prime } \\ p \neq p_{i}, q_{j}}} G_{\nu, p} \neq 0
$$


This completes the proof of the theorem.

Since, for a given $x_{0} \in \mathbb{R}$ and any $\varepsilon>0$, there exists $\mathbf{m} \in \mathbb{Z}^{\nu}$ and $n \in \mathbb{N}$ satisfying $|2 \pi| \frac{\mathbf{m}}{n}+\boldsymbol{\alpha} \mid-$

$x_{0} \mid<\varepsilon$, we may expect that the set of singularities of $\Phi(s, \boldsymbol{\alpha}, t ; \nu)$ forms a dense subset of $\mathbb{R}$. In other words, this expectation suggests the

Conjecture 2.5. The imaginary axis is a natural boundary of $L_{\sqrt{+^{2}}}(s, \boldsymbol{\alpha} ; \nu)$.

\section{Determinants of Laplacians}

As we have shown, the Ruelle type $L$-function $L_{\sqrt{+^{2}}}(s, \chi)$ (defined by the Euler product) does not seem to be extended to the entire plane $\mathbb{C}$ as a meromorphic function. Hence, in particular, it may not have a determinant expression for a "Laplacian". Thus, contrary to the discussion above, one may naturally ask the question; does the function defined by a determinant of the Laplacian on the torus $\mathbb{T}^{\nu}:=\mathbb{R}^{\nu} / \mathbb{Z}^{\nu}$ have any Euler product expression in an appropriate sense ?

We now recall the definition of the determinant of a Laplacian via zeta regularization. When the sequence $\left\{\lambda_{n}\right\}_{n=1,2, \ldots}$ consisting of the eigenvalues of some (infinite dimensional) operator $\Omega$ is given, we can define the determinant of $\Omega$ by $\operatorname{det}(\Omega):=\prod_{n=1}^{\infty} \lambda_{n}$. Here, in general, the zeta regularized product $\prod_{n=1}^{\infty} a_{n}$ of the sequence $\left\{a_{n}\right\}_{n=1,2, \ldots}$ is defined by $\prod_{n=1}^{\infty} a_{n}:=\exp \left(-\left.\frac{d}{d s} \sum_{n=1}^{\infty} a_{n}^{-s}\right|_{s=0}\right)$ when the Dirichlet series $\sum_{n=1}^{\infty} a_{n}^{-s}$ converges absolutely for $\operatorname{Re}(s) \gg 1$ and can be analytically continued to a holomorphic function around the origin $s=0$ (see [D] and also [KW2]).

Concerning the question above, our observation is as follows: Let $\boldsymbol{\alpha} \in \hat{\mathbb{Z}}^{\nu} \cong \mathbb{R}^{\nu} / \mathbb{Z}^{\nu}$. Let $\Delta_{\nu, \boldsymbol{\alpha}}:=$ $-\frac{1}{4 \pi^{2}} \sum_{j=1}^{\nu} \frac{\partial^{2}}{\partial x_{j}^{2}}$ be the Laplacian acting on the space of smooth sections of the line bundle defined by the unitary character $\chi_{\boldsymbol{\alpha}}: \mathbb{Z}^{\nu} \ni \mathbf{n} \rightarrow e^{2 \pi i \mathbf{n} \boldsymbol{\alpha}} \in U(1)$ over the torus $\mathbb{T}^{\nu}$. It is easy to see that the $L^{2}$-eigenvalue of $\Delta_{\nu, \boldsymbol{\alpha}}$ is of the form $|\mathbf{m}+\boldsymbol{\alpha}|^{2}$. Hence we have

$$
\operatorname{det}\left(\Delta_{\nu, \boldsymbol{\alpha}}+s^{2}\right)=\prod_{\mathbf{m} \in \mathbb{Z}^{\nu}}\left(|\mathbf{m}+\boldsymbol{\alpha}|^{2}+s^{2}\right) .
$$

We now show the following theorem. To simplify the description, we write $Z_{1}(s) \cong_{k} Z_{2}(s)$ for two functions $Z_{i}(s)$ when there is a polynomial $P(s)$ of degree $k$ satisfying $Z_{1}(s)=Z_{2}(s) e^{P(s)}$.

Theorem 3.1. Let $\boldsymbol{\alpha}=\left(\alpha_{1}, \ldots, \alpha_{\nu}\right) \in \hat{\mathbb{Z}}^{\nu}=\mathbb{R}^{\nu} / \mathbb{Z}^{\nu}$. Assume $\operatorname{Re}(s)>0$.

1. Suppose that $\nu=2 \ell+1(\ell \geq 0)$. Then

$$
\begin{aligned}
& \operatorname{det}\left(\Delta_{2 \ell+1, \boldsymbol{\alpha}}+s^{2}\right)=\prod_{\mathbf{m} \in \mathbb{Z}^{2 \ell+1}}\left(|\mathbf{m}+\boldsymbol{\alpha}|^{2}+s^{2}\right) \\
& \cong_{2 \ell} \exp \left(-\frac{(-2 \pi)^{\ell+1}}{(2 \ell+1) ! !} s^{2 \ell+1}-\sum_{\mathbf{n} \in \mathbb{Z}^{2 \ell+1} \backslash\{0\}} \frac{1}{|\mathbf{n}|^{\ell+1}} \sum_{k=0}^{\ell} \frac{c_{k}^{(\ell)}}{(2 \pi|\mathbf{n}|)^{k}} s^{\ell-k} e^{2 \pi i \mathbf{n} \boldsymbol{\alpha}} e^{-2 \pi|\mathbf{n}| s}\right),
\end{aligned}
$$

where the sequence $c_{k}^{(\ell)}(0 \leq k \leq \ell)$ is defined by

$$
c_{0}^{(\ell)}=1, \quad c_{k}^{(\ell)}= \begin{cases}1 & \text { if } \ell=0, \\ \frac{1}{2^{k} k !} \prod_{j=1-k}^{k}(\ell+j) & \text { if } l \geq 1, \quad(k \geq 1) .\end{cases}
$$


Moreover, when $\nu=1$ (that is, $\ell=0$ ), we have

$$
\operatorname{det}\left(\Delta_{1, \alpha}+s^{2}\right)=\prod_{m=-\infty}^{\infty}\left((m+\alpha)^{2}+s^{2}\right)=\exp \left(2 \pi s-2 \sum_{n=1}^{\infty} \frac{\cos (2 \pi n \alpha)}{n} e^{-2 \pi n s}\right) .
$$

2. Suppose that $\nu=2 \ell(\ell \geq 1)$. Then

$$
\begin{aligned}
& \operatorname{det}\left(\Delta_{2 \ell, \boldsymbol{\alpha}}+s^{2}\right)=\prod_{\mathbf{m} \in \mathbb{Z}^{2 \ell}}\left(|\mathbf{m}+\boldsymbol{\alpha}|^{2}+s^{2}\right) \\
& \quad \simeq_{2 \ell} \exp \left(\frac{4(-1)^{\ell} \pi^{\ell}}{(\ell-1) !} s^{2 \ell} \log s-4 \ell s^{\ell} \sum_{\mathbf{n} \in \mathbb{Z}^{2 \ell} \backslash\{0\}} \frac{1}{|\mathbf{n}|^{\ell}} e^{2 \pi i \mathbf{n} \boldsymbol{\alpha}} K_{\ell}(2 \pi|\mathbf{n}| s)\right),
\end{aligned}
$$

where $K_{\ell}(s)$ is the $K$-Bessel function.

Before starting the proof, we remark that the formula in the theorem can be considered as a sort of an Euler product expression of the determinant of the Laplacian. Actually, when $\nu=1$, from the identity (3.3) we easily find that

$$
\operatorname{det}\left(\Delta_{1, \alpha}+s^{2}\right)=e^{2 \pi s}\left(1-e^{2 \pi i \alpha} e^{-2 \pi s}\right)\left(1-e^{-2 \pi i \alpha} e^{-2 \pi s}\right) .
$$

Since the only primitive closed geodesic on $S^{1}=\mathbb{R} / \mathbb{Z}$ is the circle of length $2 \pi$, the right hand side can be regarded as the Euler product (over the primitive element of $\mathbb{Z}$ ). Hence it is reasonable to regard the right hand side of $(3.1) /(3.4)$ as an Euler product.

We first recall the Bessel functions. Let $J_{\lambda}(x)$ be the Bessel function of the first kind of order $\lambda$. Namely, $J_{\lambda}(x)$ satisfies the Bessel equation of order $\lambda$ :

$$
\left(\frac{d^{2}}{d x^{2}}+\frac{1}{x} \frac{d}{d x}+\left(1-\frac{\lambda^{2}}{x^{2}}\right)\right) J_{\lambda}(x)=0 .
$$

Then the $K$-Bessel function $K_{\lambda}(x)$ is defined as

$$
K_{\lambda}(x):=\frac{\pi}{2} \frac{I_{-\lambda}(x)-I_{\lambda}(x)}{\sin \pi \lambda}, \quad I_{\lambda}(x):=e^{-\lambda \pi i / 2} J_{\lambda}(i x) .
$$

The following properties are well known (See, e.g., [AAR]).

Lemma 3.2. (i) We have

$$
\int_{0}^{2 \pi} e^{i x \cos \theta} d \theta=2 \pi J_{0}(x)
$$

(ii) For $\operatorname{Re}(\xi)>-1$ and $\operatorname{Re}(\eta)>-1$, we have

$$
\frac{x^{\xi} y^{\eta} J_{\xi+\eta+1}\left(\left(x^{2}+y^{2}\right)^{1 / 2}\right)}{\left(x^{2}+y^{2}\right)^{(\xi+\eta+1) / 2}}=\int_{0}^{\pi / 2} J_{\xi}(x \sin \theta) J_{\eta}(y \cos \theta) \sin ^{\xi+1} \theta \cos ^{\eta+1} \theta d \theta .
$$

(iii) For $a, b>0$ and $-1<\operatorname{Re}(\lambda)<2 \operatorname{Re}(\mu)+3 / 2$, we have

$$
\int_{0}^{\infty} \frac{x^{\lambda+1} J_{\lambda}(b x)}{\left(x^{2}+a^{2}\right)^{\mu+1}} d x=\frac{a^{\lambda-\mu} b^{\mu}}{2^{\lambda} \Gamma(\lambda+1)} K_{\lambda-\mu}(a b),
$$


Proof of Theorem 3.1. By the definition of the regularized product, for a sufficiently large integer $j$, we have

$$
\begin{aligned}
& \frac{\partial^{j}}{\partial t^{j}} \log \left(\prod_{\mathbf{m} \in \mathbb{Z}^{\nu}}\left(|\mathbf{m}+\boldsymbol{\alpha}|^{2}+t\right)\right) \\
= & -\left.\frac{d}{d w}\left[(-1)^{j} w(w+1) \cdots(w+j-1) \sum_{\mathbf{m} \in \mathbb{Z}^{\nu}}\left(|\mathbf{m}+\boldsymbol{\alpha}|^{2}+t\right)^{-w-j}\right]\right|_{w=0} \\
= & (-1)^{j-1}(j-1) ! \sum_{\mathbf{m} \in \mathbb{Z}^{\nu}}\left(|\mathbf{m}+\boldsymbol{\alpha}|^{2}+t\right)^{-j} .
\end{aligned}
$$

Put $j=\ell+1$ for both cases $\nu=2 \ell+1$ and $2 \ell$. Since $j=\ell+1>\nu / 2$, the series in the right hand side converges absolutely. Since $\frac{\partial}{\partial t}=\frac{1}{2 s} \frac{\partial}{\partial s}$ if $t=s^{2}$, we have

$$
\left(\frac{1}{2 s} \frac{\partial}{\partial s}\right)^{\ell+1} \log \left(\prod_{\mathbf{m} \in \mathbb{Z}^{\nu}}\left(|\mathbf{m}+\boldsymbol{\alpha}|^{2}+s^{2}\right)\right)=(-1)^{\ell} \ell ! \sum_{\mathbf{m} \in \mathbb{Z}^{\nu}}\left(|\mathbf{m}+\boldsymbol{\alpha}|^{2}+s^{2}\right)^{-\ell-1} .
$$

1. The case $\nu=2 \ell+1(\ell \geq 0)$ : By the Poisson summation formula $(2.6)$ with $-\ell-1=-\frac{\nu+1}{2}$, we see that

$$
\frac{\partial}{\partial s}\left(\frac{1}{2 s} \frac{\partial}{\partial s}\right)^{\ell} \log \left(\prod_{\mathbf{m} \in \mathbb{Z}^{2 \ell+1}}\left(|\mathbf{m}+\boldsymbol{\alpha}|^{2}+s^{2}\right)\right)=2(-1)^{\ell} \pi^{\ell+1} \sum_{\mathbf{n} \in \mathbb{Z}^{2 \ell+1}} e^{2 \pi i \mathbf{n} \boldsymbol{\alpha}} e^{-2 \pi s|\mathbf{n}|} .
$$

To integrate this equation, we need the following lemma.

Lemma 3.3. Define a polynomial $P_{\ell}(s, a)(\ell=0,1,2, \ldots)$ in $s$ by

$$
P_{\ell}(s, a):= \begin{cases}\frac{2^{\ell+1}}{(2 \ell+1) ! !} s^{2 \ell+1} & \text { if } a=0, \\ \left(-\frac{2}{a}\right)^{\ell+1} \sum_{k=0}^{\ell} c_{k}^{(\ell)} a^{-k} s^{\ell-k} & \text { if } a \neq 0,\end{cases}
$$

where $c_{k}^{(\ell)}$ are given in (3.2). Then, $P_{\ell}(s, a)$ satisfies the equation

$$
\frac{\partial}{\partial s}\left(\frac{1}{2 s} \frac{\partial}{\partial s}\right)^{\ell}\left\{e^{-a s} P_{\ell}(s, a)\right\}=2 e^{-a s} .
$$

Proof. To prove (3.13), it is enough to show

$$
-a P_{\ell}(s, a)+P_{\ell}^{\prime}(s, a)=2 s P_{\ell-1}(s, a) \quad(\ell=1,2, \ldots) .
$$

Hence the case $a=0$ is clear. If $a \neq 0$, this is equivalent to the recursion

$$
c_{k}^{(\ell)}-c_{k}^{(\ell-1)}=(\ell-k+1) c_{k-1}^{(\ell)} \quad(k=1,2, \ldots, \ell) .
$$

One can easily check this recursion from the definition of $c_{k}^{(\ell)}$, whence the claim follows.

From the equation (3.12), it follows immediately that

$$
\begin{aligned}
\frac{\partial}{\partial s}\left(\frac{1}{2 s} \frac{\partial}{\partial s}\right)^{\ell} & {\left[\log \left(\prod_{\mathbf{m} \in \mathbb{Z}^{2 \ell+1}}\left(|\mathbf{m}+\boldsymbol{\alpha}|^{2}+s^{2}\right)\right)\right.} \\
& \left.-(-1)^{\ell} \pi^{\ell+1}\left\{P_{\ell}(s, 0)+\sum_{\mathbf{n} \in \mathbb{Z}^{2 \ell+1} \backslash\{\mathbf{0}\}} P_{\ell}(s, 2 \pi|\mathbf{n}|) e^{2 \pi i \mathbf{n} \boldsymbol{\alpha}} e^{-2 \pi s|\mathbf{n}|}\right\}\right]=0 .
\end{aligned}
$$


Hence the equation (3.1) follows. In particular, when $\nu=1$, the equation (3.1) becomes

$$
\prod_{m=-\infty}^{\infty}\left((m+\alpha)^{2}+s^{2}\right) \cong_{0} \exp \left(2 \pi s-2 \sum_{n=1}^{\infty} \frac{\cos (2 \pi n \alpha)}{n} e^{-2 \pi n s}\right) .
$$

In other words, there exists a constant $C(\alpha)$ such that

$$
\prod_{m=-\infty}^{\infty}\left((m+\alpha)^{2}+s^{2}\right)=C(\alpha) e^{2 \pi s}\left(1-e^{2 \pi i \alpha} e^{-2 \pi s}\right)\left(1-e^{-2 \pi i \alpha} e^{-2 \pi s}\right) .
$$

Since one knows in [KW1] that

$$
\prod_{m=0}^{\infty}\left((m+\alpha)^{2}+s^{2}\right)=\frac{2 \pi}{\Gamma(\alpha+i s) \Gamma(\alpha-i s)},
$$

the left hand side of (3.14) is calculated as

$$
\begin{aligned}
\prod_{m=0}^{\infty} & \left((m+\alpha)^{2}+s^{2}\right) \prod_{m=0}^{\infty}\left((m+1-\alpha)^{2}+s^{2}\right) \\
& =4 \pi^{2}\{\Gamma(\alpha+i s) \Gamma(1-(\alpha+i s)) \Gamma(\alpha-i s) \Gamma(1-(\alpha-i s))\}^{-1} \\
& =4 \sin \pi(\alpha+i s) \sin \pi(\alpha-i s) \\
& =e^{2 \pi s}\left(1-e^{2 \pi i \alpha} e^{-2 \pi s}\right)\left(1-e^{-2 \pi i \alpha} e^{-2 \pi s}\right) .
\end{aligned}
$$

Hence it proves $C(\alpha)=1$.

2. The case $\nu=2 \ell(\ell \geq 1)$ : We calculate the right hand side of $(3.11)$ for $\nu=2 \ell$. Notice that

$$
\left\{\left(|\mathbf{x}+\boldsymbol{\alpha}|^{2}+s^{2}\right)^{-\ell-1}\right\}^{\wedge}(\mathbf{y})=e^{-2 \pi i \boldsymbol{\alpha} \mathbf{y}} g_{\ell}(\mathbf{y}, s)
$$

where

$$
\begin{aligned}
g_{\ell}(\mathbf{y}, s): & =\int_{\mathbb{R}^{2 \ell}} \frac{e^{2 \pi i \mathbf{x y}}}{\left(|\mathbf{x}|^{2}+s^{2}\right)^{l+1}} d \mathbf{x} \\
& =\int_{\mathbb{R}^{2 \ell}} \frac{\exp \left(2 \pi i \sum_{j=1}^{\ell}\left(x_{2 j-1} y_{2 j-1}+x_{2 j} y_{2 j}\right)\right)}{\left(\sum_{j=1}^{\ell}\left(x_{2 j-1}{ }^{2}+x_{2 j}{ }^{2}\right)+s^{2}\right)^{\ell+1}} \prod_{j=1}^{\ell} d x_{2 j-1} d x_{2 j} .
\end{aligned}
$$

Introduce polar coordinates in both $\mathbf{x}, \mathbf{y} \in \mathbb{R}^{2 \ell}$ by

$$
\left\{\begin{array}{ll}
x_{2 j-1}=r_{j} \cos \theta_{j}, & x_{2 j}=r_{j} \sin \theta_{j}, \\
y_{2 j-1}=R_{j} \cos \psi_{j}, & y_{2 j}=R_{j} \sin \psi_{j}
\end{array} \quad(j=1, \ldots, \ell) .\right.
$$

Then we have

$$
\begin{aligned}
& g_{\ell}((\mathbf{R}, \boldsymbol{\psi}), s)=g_{\ell}\left(\left(R_{1} \cos \psi_{1}, R_{1} \sin \psi_{1}, \ldots, R_{\ell} \cos \psi_{\ell}, R_{\ell} \sin \psi_{\ell}\right), s\right)
\end{aligned}
$$

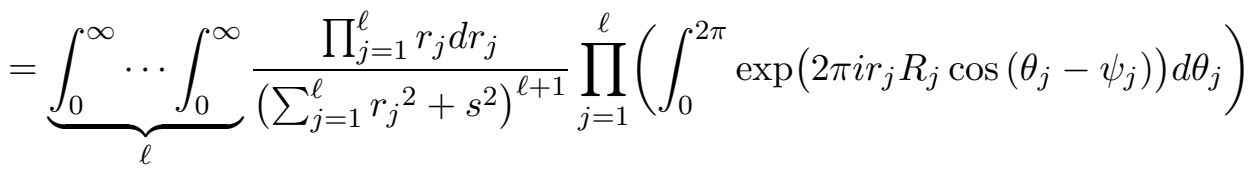

$$
\begin{aligned}
& =(2 \pi)^{\ell} \underbrace{\int_{0}^{\infty} \cdots \int_{0}^{\infty}}_{\ell} \frac{\prod_{j=1}^{\ell} r_{j} J_{0}\left(2 \pi r_{j} R_{j}\right) d r_{j}}{\left(\sum_{j=1}^{\ell} r_{j}^{2}+s^{2}\right)^{\ell+1}} .
\end{aligned}
$$


In the last equality, we have used the formula (3.8). Now it is clear that the integral $g_{\ell}((\mathbf{R}, \boldsymbol{\psi}), s)$ does not depend on the variable $\boldsymbol{\psi}=\left(\psi_{1}, \ldots, \psi_{\ell}\right)$. Hence we write $g_{\ell}((\mathbf{R}, \boldsymbol{\psi}), s)$ as $g_{\ell}(\mathbf{R}, s)$.

Lemma 3.4. For $\ell \geq 1$, we have

$$
g_{\ell}(\mathbf{R}, s)=\frac{4 \pi^{\ell+1}|\mathbf{R}| s^{-1}}{(\ell-1) !} K_{1}(2 \pi|\mathbf{R}| s),
$$

where $|\mathbf{R}|=\sqrt{R_{1}^{2}+\cdots+R_{\ell}^{2}}$.

Proof. Put $r_{j}=r\left(\prod_{k=1}^{j-1} \sin \theta_{k}\right) \cos \theta_{j}$ for $j=1, \ldots, \ell$ and $\theta_{\ell}=0$. Then one knows

$$
\sum_{j=1}^{\ell} r_{j}{ }^{2}=r^{2}, \quad \prod_{j=1}^{\ell} r_{j}=r^{\ell} \prod_{j=1}^{\ell-1} \sin ^{\ell-j} \theta_{j} \cos \theta_{j}, \quad \prod_{j=1}^{\ell} d r_{j}=r^{\ell-1} d r \prod_{j=1}^{\ell-1} \sin ^{\ell-1-j} \theta_{j} d \theta_{j} .
$$

Hence it can be written as

$$
g_{\ell}(\mathbf{R}, s)=(2 \pi)^{\ell} \int_{0}^{\infty} \frac{r^{2 \ell-1} d r}{\left(r^{2}+s^{2}\right)^{\ell+1}} I_{\ell}(r, \mathbf{R}),
$$

where $I_{0}(r, \mathbf{R}):=J_{0}(2 \pi r|\mathbf{R}|)$ and

$$
I_{\ell}(r, \mathbf{R}):=\underbrace{\int_{0}^{\pi / 2} \cdots \int_{0}^{\pi / 2}}_{\ell-1} \prod_{j=1}^{\ell} J_{0}\left(2 \pi r R_{j}\left(\prod_{k=1}^{j-1} \sin \theta_{k}\right) \cos \theta_{j}\right) \prod_{j=1}^{\ell-1} \sin ^{2(\ell-j)-1} \theta_{j} \cos \theta_{j} d \theta_{j} \quad(\ell \geq 1) .
$$

By the induction on $\ell$, it is easy to see from the formula (3.9) that

$$
I_{\ell}(r, \mathbf{R})=\frac{J_{\ell-1}(2 \pi r|\mathbf{R}|)}{(2 \pi r|\mathbf{R}|)^{\ell-1}}
$$

Hence, by using (3.10) for $a=s, b=2 \pi|\mathbf{R}|, \lambda=\ell-1$ and $\mu=\ell$, we have

$$
g_{\ell}(\mathbf{R}, s)=\frac{(2 \pi)^{\ell}}{(2 \pi|\mathbf{R}|)^{\ell-1}} \int_{0}^{\infty} \frac{r^{\ell} J_{\ell-1}(2 \pi r|\mathbf{R}|) d r}{\left(r^{2}+s^{2}\right)^{\ell+1}}=\frac{4 \pi^{\ell+1}|\mathbf{R}| s^{-1}}{(\ell-1) !} K_{-1}(2 \pi|\mathbf{R}| s) .
$$

Since $K_{-\lambda}(s)=K_{\lambda}(s)$, the lemma follows.

By (3.15), because $|\mathbf{y}|=|\mathbf{R}|$, Lemma 3.4 shows

$$
\left\{\left(|\mathbf{x}+\boldsymbol{\alpha}|^{2}+s^{2}\right)^{-\ell-1}\right\}^{\wedge}(\mathbf{y})=\frac{2 \pi^{\ell}}{(\ell-1) ! s} e^{-2 \pi i \boldsymbol{\alpha} \mathbf{y}} \cdot(2 \pi|\mathbf{y}|) K_{1}(2 \pi|\mathbf{y}| s) .
$$

Since $\lim _{s \rightarrow 0} s K_{1}(s)=1$, in particular

$$
\left\{\left(|\mathbf{x}+\boldsymbol{\alpha}|^{2}+s^{2}\right)^{-\ell-1}\right\}^{\wedge}(\mathbf{0})=\frac{2 \pi^{\ell}}{(\ell-1) ! s^{2}}
$$

The Poisson summation formula therefore yields

$$
\begin{aligned}
& \frac{\partial}{\partial s}\left(\frac{1}{2 s} \frac{\partial}{\partial s}\right)^{\ell} \log \left(\prod_{\mathbf{m} \in \mathbb{Z}^{2 \ell}}\left(|\mathbf{m}+\boldsymbol{\alpha}|^{2}+s^{2}\right)\right) \\
= & 2 s \cdot(-1)^{\ell} \ell ! \cdot \frac{2 \pi^{\ell}}{(\ell-1) ! s}\left(\frac{1}{s}+\sum_{\mathbf{n} \in \mathbb{Z}^{2 \ell} \backslash\{\mathbf{0}\}} e^{-2 \pi i \mathbf{n} \boldsymbol{\alpha}} \cdot(2 \pi|\mathbf{n}|) K_{1}(2 \pi|\mathbf{n}| s)\right) \\
= & 4 \ell(-1)^{\ell} \pi^{\ell}\left(\frac{1}{s}+\sum_{\mathbf{n} \in \mathbb{Z}^{2 \ell} \backslash\{\mathbf{0}\}} e^{2 \pi i \mathbf{n} \boldsymbol{\alpha}} \cdot(2 \pi|\mathbf{n}|) K_{1}(2 \pi|\mathbf{n}| s)\right) .
\end{aligned}
$$

In order to integrate this equation, we show the following lemma. 
Lemma 3.5. Define a function $Q_{\ell}(s, a)(\ell=0,1,2, \ldots)$ of $s$ by

$$
Q_{\ell}(s, a):= \begin{cases}\frac{1}{\ell !} s^{2 \ell} \log s & \text { if } a=0, \\ (-1)^{\ell+1}\left(2 a^{-1} s\right)^{\ell} K_{\ell}(a s) & \text { if } a \neq 0 .\end{cases}
$$

Then, $Q_{\ell}(s, a)$ satisfies the equation

$$
\frac{\partial}{\partial s}\left(\frac{1}{2 s} \frac{\partial}{\partial s}\right)^{\ell} Q_{\ell}(s, a)= \begin{cases}\frac{1}{s} & \text { if } a=0, \\ a K_{1}(a s) & \text { if } a \neq 0 .\end{cases}
$$

Proof. When $a=0$, the assertion is clear. Suppose $a \neq 0$. Since

$$
K_{\lambda}^{\prime}(s)=\frac{\lambda}{s} K_{\lambda}(s)-K_{\lambda+1}(s)=-\frac{\lambda}{s} K_{\lambda}(s)-K_{\lambda-1}(s),
$$

we notice that

$$
\begin{aligned}
\left(\frac{1}{s} \frac{\partial}{\partial s}\right)^{n}\left(s^{\lambda} K_{\lambda}(s)\right) & =(-1)^{n} s^{\lambda-n} K_{\lambda-n}(s), \\
K_{0}^{\prime}(s) & =-K_{1}(s) .
\end{aligned}
$$

Hence we have

$$
\frac{\partial}{\partial s}\left(\frac{1}{2 s} \frac{\partial}{\partial s}\right)^{\ell} Q_{\ell}(s, a)=(-1)^{\ell+1} a^{-\ell} \frac{\partial}{\partial s}\left(\frac{1}{s} \frac{\partial}{\partial s}\right)^{\ell} s^{\ell} K_{\ell}(a s) .
$$

Put $u=a s$. Since $\frac{\partial}{\partial s}=a \frac{\partial}{\partial u}$, we have

$$
\begin{aligned}
\frac{\partial}{\partial s}\left(\frac{1}{2 s} \frac{\partial}{\partial s}\right)^{\ell} Q_{\ell}(s, a) & =(-1)^{\ell+1} a^{-\ell+1} \frac{\partial}{\partial u}\left(a^{2} \frac{1}{u} \frac{\partial}{\partial u}\right)^{\ell}\left(a^{-1} u\right)^{\ell} K_{\ell}(u) \\
& =(-1)^{\ell+1} a \frac{\partial}{\partial u}\left(\frac{1}{u} \frac{\partial}{\partial u}\right)^{\ell} u^{\ell} K_{\ell}(u) \\
& =(-1)^{\ell+1} a \frac{\partial}{\partial u}(-1)^{\ell} K_{0}(u) \\
& =a K_{1}(a s) .
\end{aligned}
$$

Here we have used the equations (3.19) and (3.20).

By this lemma, it follows immediately from (3.17) that

$$
\begin{aligned}
\frac{\partial}{\partial s}\left(\frac{1}{2 s} \frac{\partial}{\partial s}\right)^{\ell} & {\left[\log \left(\prod_{\mathbf{m} \in \mathbb{Z}^{2 \ell}}\left(|\mathbf{m}+\boldsymbol{\alpha}|^{2}+s^{2}\right)\right)\right.} \\
& \left.-\left\{\frac{4(-1)^{\ell} \pi^{\ell}}{(\ell-1) !} s^{2 \ell} \log s-4 \ell s^{\ell} \sum_{\mathbf{n} \in \mathbb{Z}^{2 \ell} \backslash\{\mathbf{0}\}} \frac{1}{|\mathbf{n}|^{\ell}} e^{2 \pi i \mathbf{n} \boldsymbol{\alpha}} K_{\ell}(2 \pi|\mathbf{n}| s)\right\}\right]=0 .
\end{aligned}
$$

This completes the proof of the theorem.

Using $\sum_{\mathbf{n} \in \mathbb{Z}^{\nu} \backslash\{\mathbf{0}\}}=\sum_{n=1}^{\infty} \sum_{\mathbf{m} \in \mathbb{Z}^{\nu},|\mathbf{m}|^{2}=n}$, we immediately obtain from Theorem 3.1 the following 
Corollary 3.6. Put

$$
r_{\nu}(n, \boldsymbol{\alpha}):=\sum_{\mathbf{m} \in \mathbb{Z}^{\nu},|\mathbf{m}|^{2}=n} e^{2 \pi i \mathbf{m} \boldsymbol{\alpha}}
$$

For $\operatorname{Re}(s)>0$, we have

$$
\begin{aligned}
\operatorname{det}\left(\Delta_{2 \ell+1, \boldsymbol{\alpha}}+s^{2}\right) & \cong_{2 \ell} \exp \left(-\frac{(-2 \pi)^{\ell+1}}{(2 \ell+1) ! !} s^{2 \ell+1}-\sum_{n=1}^{\infty} \sum_{k=0}^{\ell} \frac{r_{2 \ell+1}(n, \boldsymbol{\alpha}) c_{k}^{(\ell)}}{n^{\frac{\ell+1}{2}}(2 \pi \sqrt{n})^{k}} s^{\ell-k} e^{-2 \pi \sqrt{n} s}\right), \\
\operatorname{det}\left(\Delta_{2 \ell, \boldsymbol{\alpha}}+s^{2}\right) & \cong_{2 \ell} \exp \left(\frac{4(-1)^{\ell} \pi^{\ell}}{(\ell-1) !} s^{2 \ell} \log s-4 \ell s^{\ell} \sum_{n=1}^{\infty} \frac{r_{2 \ell}(n, \boldsymbol{\alpha})}{n^{\frac{\ell}{2}}} K_{\ell}(2 \pi \sqrt{n} s)\right) .
\end{aligned}
$$

Remark 3.7. The series $r_{\nu}(n, \boldsymbol{\alpha})$ have been studied, for instance in [BB], [BD1], etc, in connection with the fluctuations of the number of lattice points inside a large sphere centered at $\boldsymbol{\alpha} \in \mathbb{R}^{\nu}$. In particular, quite recently, the limit distribution of the mean value of the square sum $X^{-\frac{\nu}{2}} \sum_{n \leq X}\left|r_{\nu}(n, \boldsymbol{\alpha})\right|^{2}$ for $X \rightarrow \infty$ has been obtained in [Ma] explicitly when $\boldsymbol{\alpha}$ satisfies a certain diophantine condition.

\section{Arithmetic functions arising from $\log L_{\sqrt{+^{2}}}(s, \alpha)$}

By the definition of $L_{\sqrt{+^{2}}}(s, \boldsymbol{\alpha} ; \nu)$ we can calculate

$$
\log L_{\sqrt{+^{2}}}(s, \boldsymbol{\alpha} ; \nu)=\sum_{\operatorname{gcd} \mathbf{p}=1} \sum_{\ell=1}^{\infty} \frac{e^{2 \pi i \ell \mathbf{p} \boldsymbol{\alpha}} e^{-s \ell|\mathbf{p}|}}{\ell}=\sum_{n=1}^{\infty} \sum_{|\mathbf{m}|^{2}=n}(\operatorname{gcd} \mathbf{m})^{-1} e^{2 \pi i \mathbf{m} \boldsymbol{\alpha}} e^{-s \sqrt{n}}
$$

Hence, if we put

$$
M_{\nu}(n, \boldsymbol{\alpha}, x)=\sum_{\mathbf{m} \in \mathbb{Z}^{\nu},|\mathbf{m}|^{2}=n}(\operatorname{gcd} \mathbf{m})^{-x} e^{2 \pi i \mathbf{m} \boldsymbol{\alpha}}
$$

we have

$$
\log L_{\sqrt{+^{2}}}(s, \boldsymbol{\alpha} ; \nu)=\sum_{n=1}^{\infty} M_{\nu}(n, \boldsymbol{\alpha}, 1) e^{-s \sqrt{n}} .
$$

We remark that $M_{\nu}(n, \boldsymbol{\alpha}, 0)=r_{\nu}(n, \boldsymbol{\alpha})$, where $r_{\nu}(n, \boldsymbol{\alpha})$ is defined in Corollary 3.6. In [KW3] we prove that $M_{\nu}(n, \mathbf{0}, x)$ is a multiplicative function (w.r.t. the variable $n$ ) when $\nu=2$ and study an asymptotic distribution of the average. The aim of this section is to generalize the results in [KW3] to the cases of $\nu=4,6$ and 8 .

We first notice that

$$
M_{\nu}(n, \boldsymbol{\alpha}, x)=\sum_{\ell=1}^{\infty} \ell^{-x} \sum_{\operatorname{gcd} \mathbf{m}=\ell,|\mathbf{m}|^{2}=n} e^{2 \pi i \mathbf{m} \boldsymbol{\alpha}}=\sum_{\ell^{2} \mid n} g_{x}\left(\ell^{2}\right) \widetilde{r}_{\nu}\left(\frac{n}{\ell^{2}}, \ell \boldsymbol{\alpha}\right),
$$

where

$$
g_{x}(n):=\left\{\begin{array}{ll}
n^{-\frac{x}{2}} & \text { if } n \text { is square, } \\
0 & \text { otherwise, }
\end{array} \quad \text { and } \quad \widetilde{r}_{\nu}(n, \boldsymbol{\alpha}):=\sum_{\substack{\mathbf{m} \in \mathbb{Z}^{\nu},|\mathbf{m}|^{2}=n \\
\text { gcd } \mathbf{m}=1}} e^{2 \pi i \mathbf{m} \boldsymbol{\alpha}}\right.
$$


We note that the function $g_{x}(n)$ is multiplicative. Hence if $\boldsymbol{\alpha}=\mathbf{0}$, we have $M_{\nu}(n, x)=g_{x} * \widetilde{r}_{\nu}(n)$ where $M_{\nu}(n, x):=M_{\nu}(n, \mathbf{0}, x)$ and $\widetilde{r}_{\nu}(n):=\widetilde{r}_{\nu}(n, \mathbf{0})$. Notice also that

$$
r_{\nu}(n, \boldsymbol{\alpha})=\sum_{\ell^{2} \mid n} \widetilde{r}_{\nu}\left(\frac{n}{\ell^{2}}, \ell \boldsymbol{\alpha}\right),
$$

Let

$$
D_{\nu}(s ; \boldsymbol{\alpha}, x):=\sum_{n=1}^{\infty} M_{\nu}(n, \boldsymbol{\alpha}, x) n^{-s}
$$

From (4.3), we have

$$
\begin{aligned}
D_{\nu}(s ; \boldsymbol{\alpha}, x) & =\sum_{n=1}^{\infty} \sum_{\ell^{2} \mid n} g_{x}\left(\ell^{2}\right) \widetilde{r}_{\nu}\left(\frac{n}{\ell^{2}}, \ell \boldsymbol{\alpha}\right) n^{-s}=\sum_{\ell=1}^{\infty} \sum_{k=1}^{\infty} g_{x}\left(\ell^{2}\right) \widetilde{r}_{\nu}\left(\frac{k \ell^{2}}{\ell^{2}}, \ell \boldsymbol{\alpha}\right)\left(k \ell^{2}\right)^{-s} \\
& =\sum_{\ell=1}^{\infty} g_{x}\left(\ell^{2}\right) \ell^{-2 s} \sum_{k=1}^{\infty} \widetilde{r}_{\nu}(k, \ell \boldsymbol{\alpha}) k^{-s} .
\end{aligned}
$$

We further define the functions

$$
\mathcal{L}_{\nu}(s ; \boldsymbol{\alpha}):=\sum_{n=1}^{\infty} r_{\nu}(n, \boldsymbol{\alpha}) n^{-s} \quad \text { and } \quad \widetilde{\mathcal{L}}_{\nu}(s ; \boldsymbol{\alpha}):=\sum_{n=1}^{\infty} \widetilde{r}_{\nu}(n, \boldsymbol{\alpha}) n^{-s}
$$

(for the study of the function $\mathcal{L}_{\nu}(s ; \mathbf{0})$, see $\left.[\mathrm{BC}]\right)$. Then we have from (4.4) and (4.5) that

$$
\mathcal{L}_{\nu}(s ; \boldsymbol{\alpha})=\sum_{\ell=1}^{\infty} \ell^{-2 s} \widetilde{\mathcal{L}}_{\nu}(s ; \ell \boldsymbol{\alpha}) \quad \text { and } \quad D_{\nu}(s ; \boldsymbol{\alpha}, x)=\sum_{\ell=1}^{\infty} g_{x}\left(\ell^{2}\right) \ell^{-2 s} \widetilde{\mathcal{L}}_{\nu}(s ; \ell \boldsymbol{\alpha}) .
$$

In particular, we put $\mathcal{L}_{\nu}(s):=\mathcal{L}_{\nu}(s ; \mathbf{0})$ and $\widetilde{\mathcal{L}}_{\nu}(s):=\widetilde{\mathcal{L}}_{\nu}(s ; \mathbf{0})$ when $\boldsymbol{\alpha}=\mathbf{0}$. Then we have

$$
\mathcal{L}_{\nu}(s)=\zeta(2 s) \widetilde{\mathcal{L}}_{\nu}(s) \quad \text { and } \quad D_{\nu}(s ; x)=\zeta(x+2 s) \widetilde{\mathcal{L}}_{\nu}(s),
$$

because

$$
\sum_{\ell=1}^{\infty} g_{x}\left(\ell^{2}\right) \ell^{-2 s}=\sum_{\ell=1}^{\infty}\left(\ell^{2}\right)^{-\frac{x}{2}} \ell^{-2 s}=\sum_{\ell=1}^{\infty} \ell^{-x-2 s}=\zeta(x+2 s) .
$$

From (4.6), we obtain

$$
D_{\nu}(s ; x)=\zeta(x+2 s) \zeta(2 s)^{-1} \mathcal{L}_{\nu}(s) .
$$

Note that $r_{\nu}(n, \mathbf{0})=r_{\nu}(n)$. Thus, a similar discussion performed in [KW3] gives the following asymptotic average of $M_{\nu}(n, \mathbf{0}, x)$ for $\nu=2,4,6$ and 8 .

Example 4.1 (The case $\boldsymbol{\nu}=\mathbf{2}$ (studied in [KW3])). From (2.13), we have

$$
\mathcal{L}_{2}(s)=4 \zeta(s) L_{-4}(s) .
$$

where $L_{-4}(s):=\sum_{n=1}^{\infty} \chi_{-4}(n) n^{-s}$. Hence it follows from (4.7) that

$$
D_{2}(s ; x)=4 \zeta(x+2 s) \zeta(2 s)^{-1} \zeta(s) L_{-4}(s) .
$$


Therefore, by the Tauberian theorem (see $[\mathrm{MM}]$ ), we obtain

$$
\sum_{n \leq X} M_{2}(n, x)= \begin{cases}\left(\frac{2 \zeta\left(\frac{-x+1}{2}\right) L_{-4}\left(\frac{-x+1}{2}\right)}{\zeta(-x+1)}+o(1)\right) X^{\frac{-x+1}{2}} & \text { if } \quad x<-1 \\ \left(\frac{3}{\pi}+o(1)\right) X \log X & \text { if } \quad x=-1 \\ \left(\frac{2 \cdot 3 \zeta(x+2)}{\pi}+o(1)\right) X & \text { if } \quad x>-1\end{cases}
$$

as $X \rightarrow \infty$, since $\zeta(2)=\pi^{2} /(2 \cdot 3)$ and $L_{-4}(1)=\pi / 2^{2}$. In particular, we have

$$
\sum_{n \leq X} M_{2}(n, 1)=\left(\frac{2 \cdot 3}{\pi} \zeta(3)+o(1)\right) X \quad(X \rightarrow \infty) .
$$

Remark 4.2. The value $6 \zeta(2+x) / \pi^{2}$ for the case of $x>-1$ in Corollary 4.4 in [KW3] is incorrect and should be $6 \zeta(2+x) / \pi$.

Remark 4.3. From the example above, we have

$$
\lim _{X \rightarrow \infty} \frac{1}{X} \sum_{n \leq X} M_{2}(n, 1)=\pi \cdot \frac{\zeta(3)}{\zeta(2)} .
$$

The appearance of the ratio of $\zeta(3) / \zeta(2)$ can be also found at the study of the mean square limit for lattice points in the 3 -dim sphere in $[\mathrm{J}]$ and $[\mathrm{BD} 2]$.

Example 4.4 (The case $\boldsymbol{\nu}=4$ ). One can calculate from (2.14) that

$$
\mathcal{L}_{4}(s)=8\left(1-4^{1-s}\right) \zeta(s) \zeta(s-1)
$$

and hence

$$
D_{4}(s ; x)=8\left(1-4^{1-s}\right) \zeta(x+2 s) \zeta(2 s)^{-1} \zeta(s) \zeta(s-1) .
$$

Therefore it holds that

$$
\sum_{n \leq X} M_{4}(n, x)= \begin{cases}\left(\frac{4\left(1-4^{\frac{x+1}{2}}\right) \zeta\left(\frac{-x+1}{2}\right) \zeta\left(\frac{-x-1}{2}\right)}{\zeta(-x+1)}+o(1)\right) X^{\frac{-x+1}{2}} & \text { if } \quad x<-3 \\ \left(\frac{3^{2} \cdot 5}{\pi^{2}}+o(1)\right) X^{2} \log X & \text { if } \quad x=-3 \\ \left(\frac{2 \cdot 3^{2} \cdot 5 \zeta(x+4)}{\pi^{2}}+o(1)\right) X^{2} & \text { if } \quad x>-3\end{cases}
$$

as $X \rightarrow \infty$, since $\zeta(4)=\pi^{4} /\left(2 \cdot 3^{2} \cdot 5\right)$. In particular, we have

$$
\sum_{n \leq X} M_{4}(n, 1)=\left(\frac{2 \cdot 3^{2} \cdot 5}{\pi^{2}} \zeta(5)+o(1)\right) X^{2} \quad(X \rightarrow \infty) .
$$

Example 4.5 (The case $\boldsymbol{\nu}=\mathbf{6}$ ). It is known (see, e.g., $[\mathrm{BC}]$ ) that

$$
\begin{aligned}
& r_{6}(n)=16 \sum_{m \mid n} \chi_{-4}\left(\frac{n}{m}\right) m^{2}-4 \sum_{m \mid n} \chi_{-4}(m) m^{2}, \\
& \mathcal{L}_{6}(s)=16 \zeta(s-2) L_{-4}(s)-4 \zeta(s) L_{-4}(s-2) .
\end{aligned}
$$


Hence we have

$$
D_{6}(s ; x)=\zeta(x+2 s) \zeta(2 s)^{-1}\left(16 \zeta(s-2) L_{-4}(s)-4 \zeta(s) L_{-4}(s-2)\right) .
$$

Therefore we obtain

$$
\sum_{n \leq X} M_{6}(n, x)= \begin{cases}\left(\frac{8 \zeta\left(\frac{-x-3}{2}\right) L_{-4}\left(\frac{-x+1}{2}\right)-2 \zeta\left(\frac{-x+1}{2}\right) L_{-4}\left(\frac{-x-3}{2}\right)}{\zeta(-x+1)}+o(1)\right) X^{\frac{-x+1}{2}} & \text { if } \quad x<-5, \\ \left(\frac{3^{3} \cdot 5 \cdot 7}{2^{2} \pi^{3}}+o(1)\right) X^{3} \log X & \text { if } \quad x=-5 \\ \left(\frac{3^{3} \cdot 5 \cdot 7 \zeta(x+6)}{2 \pi^{3}}+o(1)\right) X^{3} & \text { if } \quad x>-5\end{cases}
$$

as $X \rightarrow \infty$, since $\zeta(6)=\pi^{6} /\left(3^{3} \cdot 5 \cdot 7\right)$ and $L_{-4}(3)=\pi^{3} / 2^{5}$. In particular, we have

$$
\sum_{n \leq X} M_{6}(n, 1)=\left(\frac{3^{3} \cdot 5 \cdot 7}{2 \pi^{3}} \zeta(7)+o(1)\right) X^{3} \quad(X \rightarrow \infty) .
$$

Example 4.6 (The case $\boldsymbol{\nu}=\mathbf{8}$ ). We have from (2.15) that

$$
\mathcal{L}_{8}(s)=16\left(1-2^{1-s}+4^{2-s}\right) \zeta(s) \zeta(s-3) .
$$

Hence

$$
D_{8}(s ; x)=16\left(1-2^{1-s}+4^{2-s}\right) \zeta(x+2 s) \zeta(2 s)^{-1} \zeta(s) \zeta(s-3) .
$$

Therefore we obtain

$$
\sum_{n \leq X} M_{8}(n, x)= \begin{cases}\left(\frac{8\left(1-2^{\frac{x+1}{2}}+4^{\frac{x+3}{2}}\right) \zeta\left(\frac{-x+1}{2}\right) \zeta\left(\frac{-x-5}{2}\right)}{\zeta(-x+1)}+o(1)\right) X^{\frac{-x+1}{2}} & \text { if } \quad x<-7, \\ \left(\frac{3^{2} \cdot 5^{2} \cdot 7}{2 \pi^{4}}+o(1)\right) X^{4} \log X & \text { if } \quad x=-7, \\ \left(\frac{3^{2} \cdot 5^{2} \cdot 7 \zeta(x+8)}{\pi^{4}}+o(1)\right) X^{4} & \text { if } \quad x>-7\end{cases}
$$

as $X \rightarrow \infty$, since $\zeta(8)=\pi^{8} /\left(2 \cdot 3^{3} \cdot 5^{2} \cdot 7\right)$. In particular, we have

$$
\sum_{n \leq X} M_{8}(n, 1)=\left(\frac{3^{2} \cdot 5^{2} \cdot 7}{\pi^{4}} \zeta(9)+o(1)\right) X^{4} \quad(X \rightarrow \infty) .
$$

From the observations above, we strongly expect the following statement can be true.

Conjecture 4.7. For any $\ell \in \mathbb{N}$, there exists $\beta_{\ell} \in \mathbb{Q}$ such that

$$
\sum_{n \leq X} M_{2 \ell}(n, 1)=\left(\frac{\beta_{\ell}}{\pi^{\ell}} \zeta(2 \ell+1)+o(1)\right) X^{\ell} \quad(X \rightarrow \infty) .
$$

Additional reports (September 2007). We have obtained the conjecture above affirmatively. In fact, for any $\nu \in \mathbb{N}$, it holds that

$$
\sum_{n \leq X} M_{\nu}(n, x)= \begin{cases}\left(\frac{\mathcal{L}_{\nu}\left(\frac{1-x}{2}\right)}{\zeta(-x+1)}+o(1)\right) X^{\frac{-x+1}{2}} & \text { if } \quad x<1-\nu, \\ \left(\frac{\pi^{\frac{\nu}{2}}}{2 \zeta(\nu) \Gamma\left(\frac{\nu}{2}\right)}+o(1)\right) X^{\frac{\nu}{2}} \log X & \text { if } \quad x=1-\nu \\ \left(\frac{\pi^{\frac{\nu}{2}}}{\zeta(\nu) \Gamma\left(\frac{\nu}{2}\right)} \zeta(\nu+x)+o(1)\right) X^{\frac{\nu}{2}} & \text { if } \quad x>1-\nu\end{cases}
$$


as $X \rightarrow \infty$. In particular, for $\ell \in \mathbb{N}$, we have

$$
\begin{aligned}
\sum_{n \leq X} M_{2 \ell}(n, 1) & =\left(\frac{(-1)^{\ell+1}(2 \ell) !}{(\ell-1) ! 2^{2 \ell-1} B_{2 \ell}} \frac{\zeta(2 \ell+1)}{\pi^{\ell}}+o(1)\right) X^{\ell} \quad(X \rightarrow \infty), \\
\sum_{n \leq X} M_{2 \ell+1}(n, 1) & =\left(\frac{(-1)^{\ell} 2^{3 \ell+1} B_{2 \ell+2}}{(2 \ell-1) ! !(2 \ell+2) !} \frac{\pi^{3 \ell+2}}{\zeta(2 \ell+1)}+o(1)\right) X^{\ell+\frac{1}{2}} \quad(X \rightarrow \infty),
\end{aligned}
$$

where $B_{n}$ is the Bernoulli number (namely, the number $\beta_{\ell}$ in Conjecture 4.7 is given by $\beta_{\ell}=$ $\left.\frac{(-1)^{\ell+1}(2 \ell) !}{(\ell-1) ! 2^{2 \ell-1} B_{2 \ell}} \in \mathbb{Q}\right)$. Actually, the formula (4.8) follows from the application of the Tauberian theorem to (4.7) with $\mathcal{L}_{\nu}(s)=Z\left(s, 1_{\nu}\right)$ where $1_{\nu}$ is the identity matrix of order $\nu, Z(s, A):=\sum_{\boldsymbol{m} \in \mathbb{Z}^{\nu}} A[\boldsymbol{m}]^{-s}$ is the Epstein zeta function attached to the positive definite symmetric matrix $A$ and $A[\boldsymbol{x}]:={ }^{t} \boldsymbol{x} A \boldsymbol{x}$ for $\boldsymbol{x} \in \mathbb{R}^{\nu}$. Note that $Z(s, A)$ converges absolutely for $\operatorname{Re}(s)>\nu / 2$ and admit a meromorphic continuation to the whole plane $\mathbb{C}$ with a simple pole at $s=\nu / 2$ with residue $\pi^{\frac{\nu}{2}} /\left(\sqrt{\operatorname{det} A} \cdot \Gamma\left(\frac{\nu}{2}\right)\right)$ (see $[\mathrm{T}])$. Moreover, using $\zeta(2 n)=(-1)^{n+1} 2^{2 n-1} B_{2 n} /(2 n)$ ! for $n \in \mathbb{N}$, one can easily obtain the formulas (4.9) and (4.10) from (4.8).

\section{References}

[AAR] G.E. Andrews, R. Askey and R. Roy.: Special functions, Encyclopedia of Math. Appl., 71, Cambridge University Press, Cambridge, 1999.

[BB] P. Bleher and J. Bourgain.: Distribution of the error term for the number of lattice points inside a shifted ball, Analytic number theory, Vol. 1 (Allerton Park, IL, 1995), Progr. Math., 138, Birkhauser, Boston, MA, 1996, 141-153.

[BC] J. Borwein and K. Choi.: On Dirichlet series for sums of squares. Rankin memorial issues. Ramanujan J., 7 (2003), 95-127.

[BD1] P. Bleher and F. Dyson.: Mean square value of exponential sums related to representation of integers as sum of two squares, Acta Arith., 68 (1994), 71-84.

[BD2] P. Bleher and F. Dyson.: Mean square limit for lattice points in a sphere, Acta Arith., 68 (1994), 383-393; Erratum, ibid., 73 (1995), 199.

[D] C. Deninger.: On the $\Gamma$-factors attached to motives, Invent. Math., 104 (1991), 245-261.

[J] V. Jarník.: Über die Mittelwertsätze der Gitterpunktlehre, Časopis Pěst. Mat. Fys., 69 (1940), 148-174.

[KW1] N. Kurokawa and M. Wakayama.: A generalization of Lerch's formula, Czechoslovak Math. J., 54(129) (2004), 941-947.

[KW2] N. Kurokawa and M. Wakayama.: Zeta regularizations, "Representations of Lie groups, harmonic analysis on homogeneous spaces and quantization" (Edited by G. Van Dijk and V.F. Molchanov), Acta Appl. Math., 81 (2004), 147-166.

[KW3] N. Kurokawa and M. Wakayama.: Ruelle type zeta functions for tori and some arithmetics, Internat. J. Math., 15 (2004), 691-715. 
[L] M. Lerch.: Dalši studie v oboru Malmsténovských řad, Rozpravy České Akad., 3 (1894), no. $28,1-61$.

[Ma] J. Marklof.: Mean square value of exponential sums related to the representation of integers as sums of squares, Acta Arith., 117 (2005), 353-370.

[Mu C. Müller.: Analysis of spherical symmetries in Euclidean spaces, Applied Math. Sci., 129, Springer-Verlag, New York, 1998.

[MM] M. R. Murty and V. K. Murty.: Non-vanishing of $L$-functions and applications, Progress in Math., 157, Birkhäuser Verlag, Basel, 1997.

[Ru] D. Ruelle.: Zeta-functions for expanding maps and Anosov flows, Invent. Math., 34 (1976), 231-242.

[T] A. Terras.: Harmonic analysis on symmetric spaces and applications. I, Springer-Verlag, New York, 1985.

\section{Nobushige KUROKAWA}

Department of Mathematics, Tokyo Institute of Technology.

Oh-okayama Meguro, Tokyo, 152-0033 JAPAN.

kurokawa@math.titech.ac.jp

MASATO WAKAYAMA

Faculty of Mathematics, Kyushu University.

Hakozaki, Fukuoka, 812-8581 JAPAN.

wakayama@math.kyushu-u.ac.jp

YOSHINORI YAMASAKI

Graduate School of Mathematics, Kyushu University.

Hakozaki, Fukuoka, 812-8581 JAPAN.

yamasaki@math.kyushu-u.ac.jp 